\title{
Intracranial thrombosis of the internal carotid artery after closed head injury
}

\author{
F. L. MASTAGLIA ${ }^{1}$, S. SAVAS, AND B. A. KAKULAS \\ From the Department of Pathology, University of Western Australia, and the Neuropathology Laboratory, \\ Royal Perth Hospital
}

Occlusion of the internal carotid artery may arise in several ways. In adults it is usually the result of thrombosis complicating atherosclerosis (Fisher, 1951, 1954; Luessenhop, 1959), whereas in children and young adults traumatic thrombosis accounts for a greater number of cases (Humphrey and Newton, 1950; Frantzen, Jacobsen, and Therkelsen, 1961; Pitner, 1966). Thrombosis of the internal carotid artery has been reported after penetrating neck injuries (Makins, 1916; Colledge and Dunn, 1917; Caldwell and Hadden, 1948; Lawrence, Shefts, and McDaniel, 1948;Raney, 1948; Hughes and Brownell, 1968), surgical operations on the neck (Fisher, 1951; Ralph, 1954), and intra-oral trauma (Pitner, 1966). In addition, it is being reported with increasing frequency as a complication of closed trauma to the head and neck (Fisher and Friedmann, 1959; Caldwell and Hadden, 1948; Schneider and Lemmen, 1952; Clarke, Dickson, and Smith, 1955; Paillas, 1955; Sedzimir, 1955; Murray, 1957; Hockaday, 1959; Luessenhop, 1959; Murphey and Miller, 1959; Verbiest and Calliauw, 1959; Gurdjian, Hardy, Lindner, and Thomas, 1963; Therkelsen and Hornnes, 1963; Thompson, 1963; Houck, Jackson, Odom, and Young, 1964; Toakley and McCaffrey, 1965; Beraud, Bilodeau, Sirois, Reinhardt, and Cote, 1966; Miller and Ayers, 1967; Peerless, Hoffman, and Hendrick, 1967; Hughes and Brownell, 1968). The majority of these reports are of thrombosis of the vessel in the neck. Although thrombosis of the intracranial portion of the internal carotid artery may occur in association with basal skull fractures (Thompson, 1963), it more commonly follows relatively minor head injuries. Diagnosis is then often difficult and the outcome is usually fatal as the occlusion is surgically inaccessible. The two cases presented illustrate the diagnostic and therapeutic difficulties and the poor prognosis in this situation.

\footnotetext{
1 Present address: Regional Neurological Centre, The General Hospital, Newcastle upon Tyne, England.
}

\section{CASE REPORTS}

CASE 1 (R.P.H. no. 131674) A.S. was a 39-year-old woman who was admitted to the Royal Perth Hospital on 29 December 1965 after a collision in a motor vehicle in which she was a passenger. She sustained a head injury and was unconscious for a few minutes. She had no recall for the accident but there was no retrograde amnesia. She had been quite well before the accident and had not been taking oral contraceptives. When examined, a scalp laceration was present in the occipital region and there were several contusions in the tissues of the right arm and left leg. She was conscious and fully oriented. The pupils reacted normally to light and the fundi were normal. There were no other cranial nerve abnormalities and no lateralizing neurological signs. The pulse was 72 per minute and the blood pressure $120 / 80 \mathrm{~mm} \mathrm{Hg}$. There were no cardiac abnormalities.

On the sixth hospital day she became drowsy, complained of severe headache and nausea, and vomited several times. Over the next 24 hours she became extremely restless and irritable. On the following day a grand-mal seizure occurred and three further seizures ensued in the next three hours. There was right-sided papilloedema and the pupils were equal and reacted normally to light. Post-ictally a flaccid right hemiparesis was present. The deep tendon reflexes were symmetrical and the plantar reflex was flexor on both sides. The pulse rate was 60 per minute and the blood pressure $110 / 60 \mathrm{~mm}$ $\mathrm{Hg}$. Intramuscular phenytoin and paraldehyde were administered and no further seizures occurred. One hour later she was still drowsy and the right pupil was larger than the left and reacted only sluggishly to light. Left carotid arteriography showed complete obstruction of the internal carotid artery in the carotid canal (Fig. 1). Her conscious state continued to deteriorate and she became deeply comatose. The pulse rate varied between 40 and 60 per minute. The left pupil became widely dilated and fixed. The right pupil was small and reacted slightly to light. Extensor spasms of all four limbs occurred. On the eighth hospital day burr holes were placed in the temporal and parietal regions on both sides. On opening the dura the brain was found to be swollen and herniated through the burr holes, particularly on the left side. Despite the administration of intravenous mannitol her condition deteriorated further and she died on the ninth hospital day. 


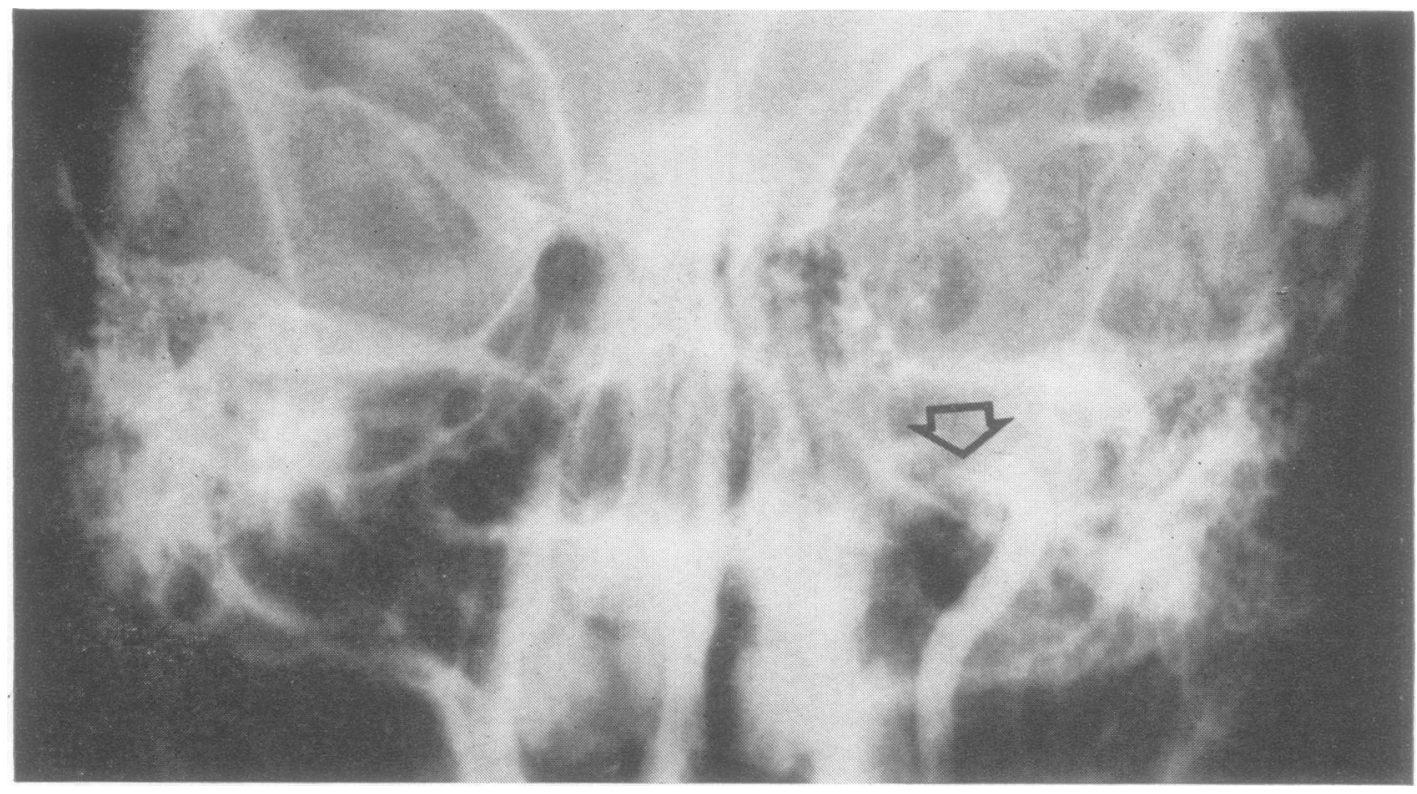

FIG. 1. Early arterial phase of left carotid arteriogram in case 1 showing complete block of the internal carotid artery iR the carotid canal (arrow).

Pathological findings (B66.71) The necropsy was performed by the Coroner's medical officer (Dr. William Laurie) who found evidence of purulent bronchitis and basal pulmonary congestion. No evidence of thrombosis was found in the veins of the lower limbs or pelvis or in the heart or aorta. There was no defect in the atrial septum. No cranial fracture was present. The brain weighed 1,310 g. Both cerebral hemispheres were swollen, the left being larger than the right. There was compression and

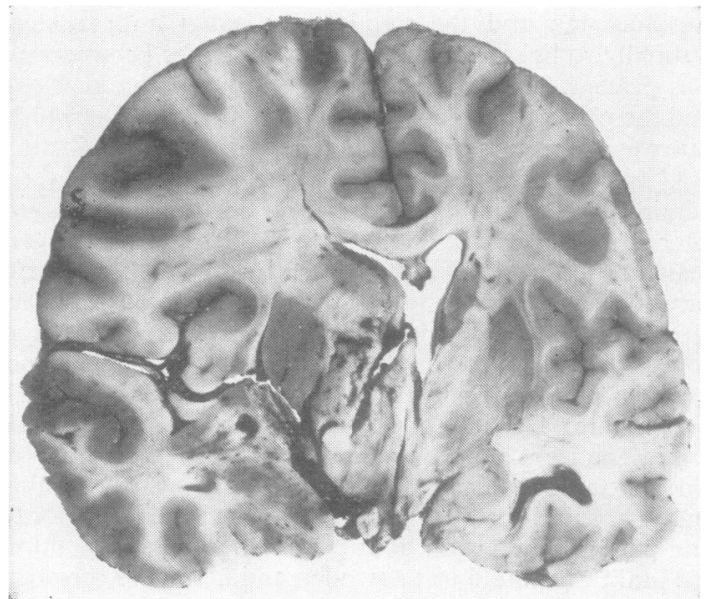

FIG. 2. Coronal section through the cerebral hemispheres in case 1 showing extensive infarction of the left hemisphere. distortion of the midbrain with extensive secondary haemorrhages in the midbrain and upper pons. The tonsils of the cerebellum were excessively grooved. The greateo part of the left cerebral hemisphere was softened. CoronaR sections through the hemispheres showed swelling and discoloration of the left frontal, temporal and parieta $\frac{8}{8}$ cortex, white matter and basal ganglia in the superficial and deep territories of supply of the left middle cerebral artery. The territory of the anterior cerebral artery was spared (Fig. 2). The inferior temporal and occipital cortex in the territory of supply of the left posterior cerebral artery was also softened and there were multiple punctate haemorrhages throughout the infarcted grey matter. Sections from the infarcted areas showed neuronal loss and the surviving neurones were shrunken and eosinophilic and showed nuclear pyknosis. Pleomorphic microgliacytes were present and small vessels were occluded by eosinophilic hyaline material. In the occipital cortex there were also many small perivascular and parenchymal haemorrhages.

Cerebral blood vessels The internal carotid arteries were patent throughout their course in the neck. However, the left internal carotid artery in the carotid canal was completely occluded by adherent laminated red and yellow thrombus which was propagated distally to the point of bifurcation of the vessel into anterior and middle cerebral arteries (Fig. 3). Microscopic examination of the thrombus showed a typical red cell and fibrin structure. The vessel wall was normal. The arteries which formed the circle of Willis were free of atheroma and their anatomical pattern was normal. The cerebral veins and venous sinuses were normal. 


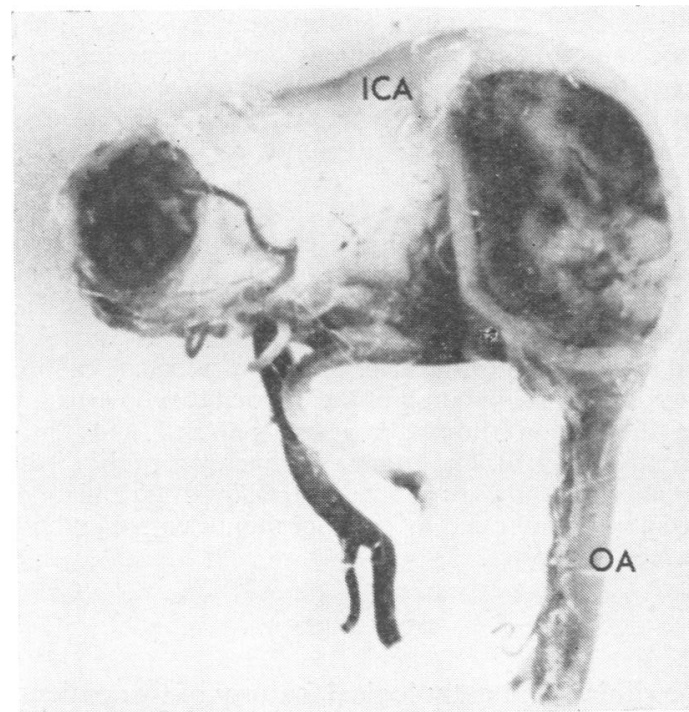

FIG. 3. Left internal carotid artery from the region of the siphon in case 1 showing complete occlusion by thrombus. $I C A=$ internal carotid artery. $O A=$ ophthalmic artery.

CASE 2 (R.P.H. no. 89669) J.S. was a previously well 39-year-old man who was admitted to the Royal Perth Hospital on 10 June 1967 after a motor vehicle accident. He was fully conscious but could not give an account of the collision. On physical examination there was a large haematoma at the angle of the left mandible, a fracture of the left clavicle, and several skin lacerations in the limbs. There were no abnormal neurological signs. His conscious state subsequently deteriorated and three hours after admission (six hours after the injury) he responded to painful stimuli only by moving the left side of the body. The right arm and leg were flaccid and the tendon jerks were diminished. The superficial abdominal reflexes were absent on the right side and the right plantar reflex was extensor. The lower half of the right side of the face failed to move in response to painful stimuli. The right pupil was slightly larger than the left, but both reacted to light both directly and consensually. The pulse rate was 80 per minute and the blood pressure $170 / 110 \mathrm{~mm} \mathrm{Hg}$. There were no cardiac abnormalities.

Radiographs of the skull showed no cranial fracture, but there was a fracture at the angle of the left mandible. Carotid arteriography performed six hours after admission showed that the left internal carotid artery was fully patent to the level of the cavernous sinus, but only a thin streak of contrast was present beyond this point. There was no filling of the anterior or middle cerebral arteries (Fig. 4). Intramuscular dexamethasone and intravenous heparin were administered at eight-hourly intervals. Twelve hours later the left pupil was fully dilated and failed to respond to light. The right pupil was constricted but reacted to light. Bilateral papilloedema was present. Shortly afterwards the right pupil also became dilated and unreactive. The blood pressure began to rise, respirations were periodic, and the plantar reflex was extensor on both sides. Burr holes were placed in the left temporal and parietal regions. The brain was found to be swollen and under increased tension. Despite intravenous infusions of

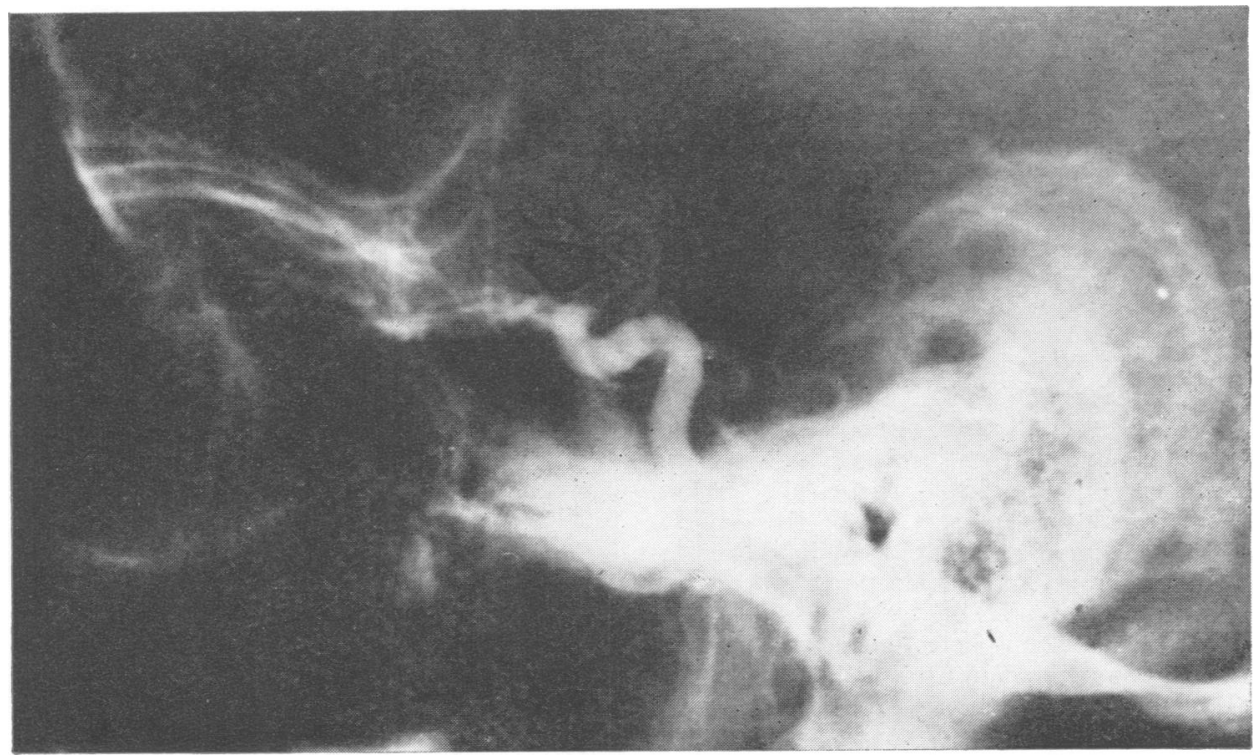

FIG. 4. Arterial phase of left carotid arteriogram in case 2 showing hold-up of contrast in the carotid siphon immediately beyond the origin of the ophthalmic artery (arrow). 


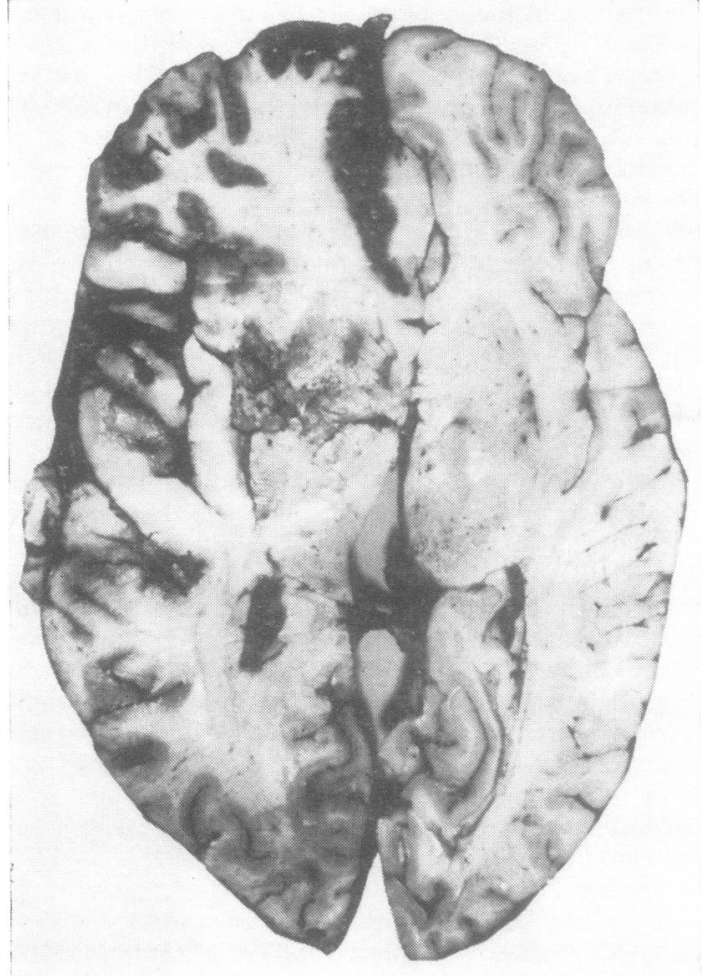

FIG. 5. Horizontal section through the cerebral hemisphere in case 2 showing haemorrhagic infarction of left frontal cortex and anaemic infarction of left temporo-parietal cortex and basal ganglia.

mannitol, the patient's condition failed to improve and he died 40 hours after admission.

Pathological findings (B67.1814) The necropsy was performed by the Coroner's medical officer (Dr. Donald Hainsworth). Necropsy findings included fractures of the left mandible and clavicle and basal pulmonary congestion and oedema. No source of emboli was found and the heart was normal. There were no skull fractures. The brain weighed $1,450 \mathrm{~g}$. The left cerebral hemisphere was swollen and there was softening and discoloration of the frontal lobe and greater part of the temporal and parietal lobes. Midline structures were displaced to the right. Both unci were excessively grooved, particularly the left. A 'Kernohan's notch' was present in the left cerebral peduncle and there were extensive secondary haemorrhages in the midbrain and upper pons. There was excessive grooving of the cerebellar tonsils. Serial coronal sections of the cerebral hemispheres showed swelling and discoloration of the cortex and basal ganglia of the left hemisphere in the anterior and middle cerebral artery territories of supply with sparing of the posterior cerebral artery territory. The infarcted frontal grey matter was haemorrhagic (Fig. 5). Microscopic examination showed excessive eosinophilia and shrinkage of neurones with loss of Nissl's substance and nuclear pyknosis. Small vessels in the infarcted frontal cortex were intensely congested and there were many perivascular and parenchymal haemorrhages. There was early emigration of polymorphonuclear leucocytes into the infarcted tissues but no glial reaction.

Cerebral blood vessels The internal carotid arteries were patent in the neck but the left internal carotid artery was completely occluded by thrombus at its point of bifurcation into anterior and middle cerebral arteries. The major vessels of the circle of Willis were free of atheroma and the communicating arteries were of adequate calibre. Microscopic examination of the internal carotid artery at the bifurcation showed a recent red cell and fibrin thrombus (Fig. 6). The intima and inner part of the media of the vessel wall were intact, but the adventitia and outer media were infiltrated by polymorphonuclear and mononuclear leucocytes.

\section{DISCUSSION}

The clinical and pathological features of two patients who died from traumatic occlusion of the intracranial portion of the internal carotid artery are reported. Both were adults in the fourth decade of life. Thrombosis followed a mild concussive head injury in both patients and in neither was there fracture o the skull. After the initial transient loss of conscious

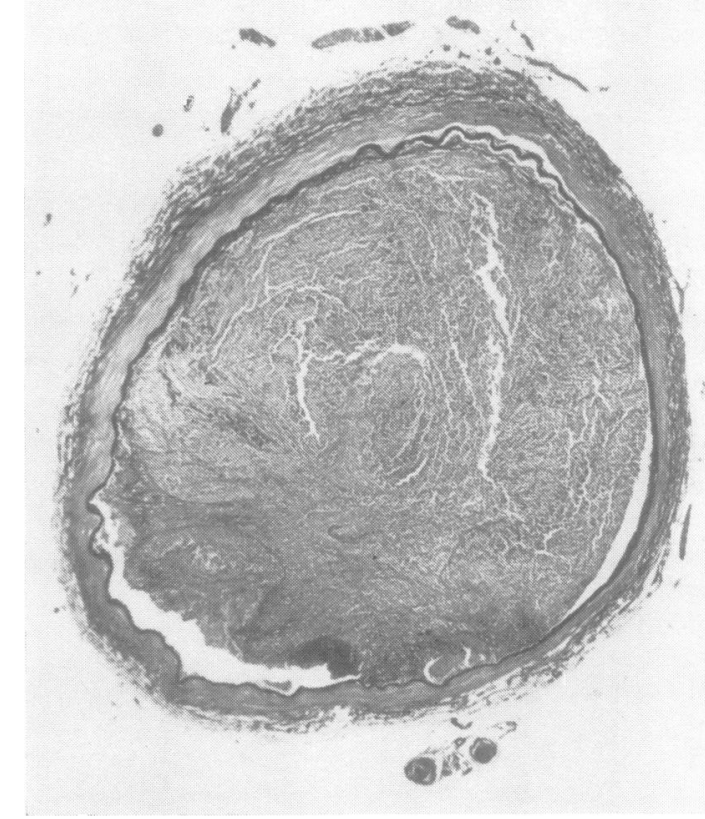

FIG. 6. Section through the terminal portion of the left internal carotid artery in case 2 showing occlusion by recent thrombus. Verhof van Giesen, $\times 75$.

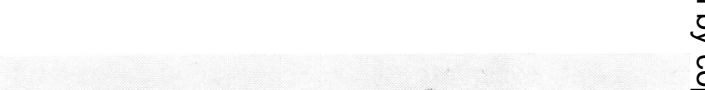


ness, there was a latent interval of six days in case 1 and six hours in case 2 , during which time the patients' neurological state was normal. In most of the previously reported cases of thrombosis of the cervical portion of the vessel (Hughes and Brownell, 1968) the occlusion was manifest within 24 hours of the initial injury. However, longer latent intervals of two to five days were described by Sedzimir (1955) in three cases of thrombosis of the intracranial portion of the vessel. Headaches and epileptic seizures were the initial manifestations in case 1 , while in case 2 deterioration in conscious state was the first indication of the occlusion. In both cases infarction of the dominant hemisphere resulted in hemiplegia, and death due to cerebral swelling and tentorial herniation occurred three days and 36 hours respectively after the appearance of neurological signs.

Several theories have been advanced to account for the occurrence of ischaemic necrosis after acute carotid occlusion in patients with an anatomically normal circle of Willis uncompromised by atheroma (Paillas, 1955; Beraud et al., 1966). Propagation of the thrombus into the middle and anterior cerebral arteries has been suggested, but this did not occur in either of the present cases. Embolization into the anterior and middle cerebral arteries from the site of primary thrombosis is considered by Pitner (1966) to be the most likely explanation and this could account for the delayed onset of symptoms in most cases. The detection of emboli at post-mortem examination of the cerebral vessels is notoriously difficult, but the finding of haemorrhagic infarction is suggestive of an embolic occlusion even when the embolus is not found. Embolization of thrombotic material from the internal carotid artery may have occurred in case 2 , in which haemorrhagic infarction was present in the frontal cortex. The other mechanisms postulated are reflex spasm of cerebral vessels due to irritation of the adventitia of the thrombosed carotid, and inadequacy of anastomotic channels. The importance of the first mechanism remains speculative, as the demonstration of arterial spasm in such cases would require contralateral angiography with adequate cross-circulation studies. The most likely mechanism would appear to be an overall inadequacy of collateral blood flow. In contrast with the often fatal outcome in cases of acute carotid occlusion, it is well recognized that gradual occlusion of one or both of these vessels may be completely compensated for by the development of an adequate collateral blood flow. In such cases, well-developed anastomoses between branches of the internal and external carotid arteries are found. In the acute situation such anastomoses are not fully developed and blood flow to the territory of the occluded vessel falls below critical levels.
The mechanism of the thrombosis itself remains obscure in most cases. There are several reports of intimal tears (Schneider and Lemmen, 1952; Verbiest and Calliauw, 1959; Gurdjian et al., 1963; Hughes and Brownell, 1968; Pearce, 1969) with associated damage to the media, curling up of these layers and eventual thrombosis. Pitner (1956) has postulated that a blunt blow to the artery produces a rapid compression and rebound of the vessel which may result in intimal disruption. Others have stressed the importance of contusion of the vessel wall, subintimal haemorrhage in the presence of atheromatous disease of the vessel, dissection of the vessel wall after intimal damage (Gurdjian et al., 1963), arterial spasm (Schneider and Lemmen, 1952), and compression of the internal carotid artery against the lateral mass of the second cervical vertebra (Peerless et al., 1967). More specifically with regard to thrombosis of the intracranial portion of the vessel, Sedzimir (1955) has suggested that, when there is relative movement of the brain in an anteroposterior direction within the confines of the skull, the artery is subject to shearing strains at the point where it leaves the cavernous sinus where it is anchored by a firm dural attachment. Such strains may lead to temporary distortion of the vessel wall with intimal damage and spasm, which favours thrombosis. The presence of an inflammatory cellular infiltrate in the adventitia and outer media of the vessel wall at the site of occlusion in case 2 may be significant in this regard. The possible contributory role of posttraumatic thrombocytosis and increased platelet adhesiveness has not been studied. It is probable that the formation of the thrombus is a gradual process and that clinical manifestations occur only when there has been significant reduction in size of the lumen of the vessel, thus accounting for the lucid interval.

The gravity of the prognosis in such cases reflects the inadequacy of present therapeutic measures. Best results have been obtained in cases of occlusion of the cervical portion of the vessel when the diagnosis is established early by arteriography and prompt surgical exploration is carried out (Gurdjian et al., 1963; Houck et al., 1964) before the changes in the brain becomes irreversible. When the occlusion is surgically inaccessible, conservative measures are usually ineffective. These include the use of parenteral vasodilators such as procaine and nicotinic acid, and stellate ganglion block aimed at reducing arterial spasm and increasing blood flow through collateral channels. The use of mannitol infusion and parenteral dexamethasone failed to influence the clinical course in the present cases. In case 2 anticoagulation was also ineffective. Such therapy may prevent propagation of thrombus into the branches of the internal 
carotid artery, but Beraud et al. (1966) have drawn attention to the risk of cerebral haemorrhage when infarction has already occurred. The anticoagulants may have contributed to the development of haemorrhagic infarction in case 2 .

\section{SUMMARY}

Thrombosis of the internal carotid artery is an uncommon but recognized complication of trauma to the head and neck. The clinical and pathological features of two fatal cases of thrombosis of the intracranial portion of the internal carotid artery after minor concussive head injuries are described. The mechanism of thrombosis and the factors responsible for the development of cerebral infarction are considered.

We wish to thank Mr. J. Grummette who prepared the photographs and the Coroner's medical officers-Dr. W. Laurie and Dr. D. Hainsworth-for submitting the brains for examination. Dr. F. L. Mastaglia was in receipt of a Saw Medical Research Fellowship from the University of Western Australia.

\section{REFERENCES}

Beraud, R., Bilodeau, B., Sirois, J., Reinhardt, G., and Cote, J. (1966). Les thromboses traumatiques de la carotide interne. Canad. med. Ass. J., 94, 537-541.

Cald well, H. W., and Hadden, F. C. (1948). Carotid artery thrombosis: Report of eight cases due to trauma. Ann. intern. Med., 28, $1132-1142$.

Clarke, P. R. R., Dickson, J., and Smith, B. J. (1955). Traumatic thrombosis of internal carotid artery following non-penetrating injury and leading to infarction of the brain. Brit. J. Surg., 43, 215-216.

Colledge, L., and Dunn, J. S. (1917). Four cases of hemiplegia caused by embolism following gunshot wounds of the carotid arteries. Lancet, 1, 57-59.

Fisher, C. M. (1951). Occlusion of the internal carotid artery. Arch. Neurol. Psychiat. (Chic.), 65, 346-377.

(1954). Occlusion of the carotid arteries. Further experisnces. Ibid., 72, 187-204.

Fisher, R. G., and Friedmann, K. R. (1959). Carotid artery thrombosis in persons 15 years of age or younger. J. Amer. med. Ass., 170, 1918-1919.

Frantzen, E., Jacobsen, H. H., and Therkelsen, J. (1961). Cerebral artery occlusions in children due to trauma to head and neck: Report of six cases verified by cerebral angiography. Neurology (Minneap.), 11, 695-700.

Gurdjian, E. S., Hardy, W. G., Lindner, D. W., and Thomas, L. M. (1963). Closed cervical cranial trauma associated with involvement of carotid and vertebral arteries. J. Neurosurg., 20, 418-427.
Hockaday, T. D. R. (1959). Traumatic thrombosis of the internal carotid artery. J. Neurol. Neurosurg. Psychiat., 22, 229-231.

Houck, W. S., Jackson, J. R., Odom, G. L., and Young, W. G. (1964). Occlusion of the internal carotid artery in the neck secondary to closed trauma to the head and neck. Ann. Surg., 159, 219-221.

Hughes, J. T., and Brownell, B. (1968). Traumatic thrombosis of the internal carotid artery in the neck. J. Neurol. Neurosurg. Psychiat., 31, 307-314.

Humphrey, J. G., and Newton, T. H. (1960). Internal carotid artery occlusion in young adults. Brain, 83, 565-578.

Lawrence, K. B., Shefts, L. M., and McDaniel, J. R. (1948). Wounds of common carotid arteries. Report of 17 cases from World War II. Amer. J. Surg. n.s. 76, 29-37.

Luessenhop, A. J. (1959). Occlusive disease of the carotid artery. Observations on the prognosis and surgical treatment. $J$. Neurosurg., 16, 705-730.

Makins, G. H. (1916). A series of cases of cerebral embolism consequent on the reception of gunshot injury to the carotid arterics. Lancet, 2, 543-546.

Miller, J. D. R., and Ayers, T. N. (1967). Post-traumatic changes in the internal carotid artery and its branches. An arteriographic study. Radiology, 89, 95-100.

Murphey, F., and Miller, J. H. (1959). Carotid insufficiency. Diagnosis and surgical treatment. J. Neurosurg., 16, 1-23.

Murray, D. S. (1957). Post-traumatic thrombosis of the internal carotid and vertebral arteries after non-penetrating injuries of the ncck. Brit. J. Surg., 44, 556-561.

Paillas, J. E. (1955). Les thromboses de la carotide interne et de ses branches. Rapports présentés à la réunion annuelle de la Société de Neurochirurgie de Langue Française, Masson et Cie, Paris.

Pearce, G. W. (1969). Personal communication.

Peerless, S. J., Hoffman, H. J., and Hendrick, E. B. (1967). Traumatict thrombosis of the internal carotid artery. Presented at the Second Canadian Congress of Neurological Sciences, 1967 Abstracted in Canad. med. Ass. J., 97, 247.

Pitner, S. E. (1966). Carotid thrombosis due to intra-oral trauma: Am unusual complication of a common childhood accident. New Engl. J. Med., 274, 764-767.

Ralph, L. L. (1954). Spontaneous thrombosis of the internal carotio artery. J. Fac. Radiol. (Lond.), 5, 210-217.

Raney, A. A. (1948). Cerebral embolism following minor wounds o the carotid artery. Report of an autopsy. Arch. Neurol. Psychiat. (Chic.) 60, 425-439.

Schneider, R. C., and Lemmen, L. J. (1952). Traumatic internal carotid artery thrombosis secondary to non-penetrating injuries to the neck. A problem in the differential diagnosis of craniocerebral trauma. J. Neurosurg., 9, 495-507.

Sedzimir, C. B. (1955). Head injury as a cause of internal carotid thrombosis. J. Neurol. Neurosurg. Psychiat., 18, 293-296.

Therkelsen, J., and Hornnes, J. (1963). Traumatic occlusion of the internal carotid artery in a child. Restored circulation by means of thrombectomy. Circulation, 28, 101-104.

Thompson, J. L. G. (1963). Traumatic thrombosis of the internal carotid artery in the carotid canal. Brit. J. Radiol., 36, 840-842.

Toakley, G., and McCaffrey J. (1965). Traumatic thrombosis of the internal carotid artery. Aust. N.Z.J. Surg., 34, 261-264.

Verbiest, H., and Calliauw, L. (1969). Direct and indirect injuries of the cervical carotid arteries. A contribution to the differential diagnosis of the post-traumatic lucid interval. Folia psychiat. neerl., 62, 371-382. 